INPLASY

PROTOCOL

To cite: Lin et al. Association of antenatal corticosteroids with morbidity and mortality among multiple gestations: a protocol for meta-analysis. Inplasy protocol 2020100105. doi: 10.37766/inplasy2020.10.0105

Received: 27 October 2020

Published: 27 October 2020

Corresponding author:

Zhengping Liu

liuzphlk81@outlook.com

Author Affiliation:

Southern Medical University

Affiliated Maternal \& Child Health Hospital of Foshan, Guangdong, China

Support: None.

Review Stage at time of this submission: Data analysis.

Conflicts of interest:

None.

\section{Association of antenatal corticosteroids with morbidity and mortality among multiple gestations: a protocol for meta-analysis}

Lin, D1; Fan, D2; Chen, G33; Luo, C4; Guo, X5; Liu, Z6.

Review question / Objective: Does antenatal corticosteroids (ACS) have benefits for reducing adverse outcomes among preterm multiple gestations?

Condition being studied: Antenatal corticosteroids, preterm multiple pregnancies and perinatal outcomes.

Information sources: The electronic databases included the PubMed, Embase, Web of Science and Cochrane Library databases.

INPLASY registration number: This protocol was registered with the International Platform of Registered Systematic Review and Meta-Analysis Protocols (INPLASY) on 27 October 2020 and was last updated on 27 October 2020 (registration number INPLASY2020100105).

\section{INTRODUCTION}

Review question / Objective: Does antenatal corticosteroids (ACS) have benefits for reducing adverse outcomes among preterm multiple gestations?

Rationale: There remains a controversy regarding the effectiveness of ACS among multiple pregnancies when using the regime as same as for singletons. From the view of pharmacokinetics, shorter half-life and faster clearance of corticosteroids in multiple pregnancies might theoretically result in reduced effectiveness of ACS among multiple pregnancies compared with singletons. The current evidence in multiple gestations is limited and less consistent. 
Condition being studied: Antenatal corticosteroids, preterm multiple pregnancies and perinatal outcomes

\section{METHODS}

Search strategy: A systematic literature search was performed by searching the PubMed, Embase, Web of Science and Cochrane Library databases. Search terms included corticosteroids, preterm, antenatal and multiple pregnancies and their synonyms.

Participant or population: Preterm multiple pregnancies.

Intervention: The intervention is antenatal corticosteroids (ACS). Complete ACS was defined as four 6-mg doses of intramuscular dexamethasone at 12-hour intervals or two 12-mg doses of intramuscular betamethasone at 24-hour intervals.

\section{Comparator: No ACS treatments.}

Study designs to be included: Observational studies (including casecontrol studies and cohort studies) and randomized controlled trial.

Eligibility criteria: Studies evaluating perinatal outcomes among preterm multiple pregnancies after use of ACS, compared with a control group without ACS treatments.

Information sources: The electronic databases included the PubMed, Embase, Web of Science and Cochrane Library databases.

Main outcome(s): The primary outcome was respiratory distress syndrome (RDS).

Additional outcome(s): Secondary outcomes included mortality, intraventricular hemorrhage (IVH), periventricular leukomalacia (PVL), necrotizing enterocolitis (NEC), retinopathy of prematurity (ROP) and bronchopulmonary dysplasia (BPD).
Data management: The following information regarding study characteristics and primary results was extracted by using a predesigned form: the first author, year of publication, country, study design, sample size, population characteristics, exclusion criteria, details of ACS administration, outcomes reported, incidence or adjusted odds ratios (aORs) of each outcome. Data extraction were independently performed by two authors and any disagreements were resolved by discussion with a third author.

Quality assessment / Risk of bias analysis: The risk of bias of the included studies was assessed based on the Newcastle-Ottawa Scale (NOS) (http://www.ohri.ca/programs/ clinical_epidemiology/oxford.asp), which consists of eight items for three subscales (4 items for selection, 1 item for comparability and 3 items for outcome).

Strategy of data synthesis: The metaanalysis was performed by using Stata 15.1 software (Stata Crop, College Station, TX). A fixed effects model was used to calculate a pooled OR if a study only provided results on subgroups and this pooled OR was used for meta-analysis. A fixed effects model with the Mantel-Haenszel method was utilized to calculate the pooled ORs and 95\% confidential intervals (CIs). When significant heterogeneity across the included studies was observed, based on the Cochran Q test (P50\%), a random effects model was utilized instead.

Subgroup analysis: Subgroup analyses were performed based on ACS completeness and administration-todelivery interval.

Sensibility analysis: Sensitivity analyses were carried out by omitting individual studies at a time.

Language: English.

Country(ies) involved: China.

Keywords: Antenatal corticosteroids; Multiple pregnancies; Respiratory distress syndrome; Meta-analysis. 
Contributions of each author:

Author 1 - Dongxin Lin conceived the study, performed analysis and drafted the manuscript.

Author 2 - Dazhi Fan selected studies and collected data.

Author 3 - Gengdong Chen selected studies and collected data.

Author 4 - Caihong Luo conceived the study and interpreted the results.

Author 5 - Xiaoling Guo interpreted the results.

Author 6 - Zhengping Liu solved the disagreements in study selection and data extraction and revised the manuscript. 